\title{
Closed-Form Green's Function Representations in Cylindrically Stratified Media for Method of Moments Applications
}

\author{
S. Karan, V. B. Ertürk, Member, IEEE, and A. Altintas, Senior Member, IEEE
}

\begin{abstract}
Closed-form Green's function (CFGF) representations for cylindrically stratified media, which can be used as the kernel of an electric field integral equation, are developed. The developed CFGF representations can safely be used in a method of moments solution procedure, as they are valid for almost all possible source and field points that lie on the same radial distance from the axis of the cylinder (such as the air-dielectric and dielectric-dielectric interfaces) including the axial line ( $\rho=\rho^{\prime}$ and $\phi=\phi^{\prime}$ ), which has not been available before. In the course of obtaining these expressions, the conventional spectral domain Green's function representations are rewritten in a different form so that i) we can attack the axial line problem and ii) the method can handle electrically large cylinders. Available acceleration techniques that exist in the literature are implemented to perform the summation over the cylindrical eigenmodes efficiently. Lastly, the resulting expressions are transformed to the spatial domain using the discrete complex image method with the help of the generalized pencil of function method, where a modified two-level approach is used. Numerical results are presented in the form of mutual coupling between two current modes to assess the accuracy of the final spatial domain CFGF representations.
\end{abstract}

Index Terms-Closed-form Green's functions, discrete complex image method (DCIM), generalized pencil of function (GPOF) method, method of moments (MoM).

\section{INTRODUCTION}

$\mathbf{T}$ HE use of closed-form Green's functions (CFGF) obtained using the discrete complex image method (DCIM) is very common for the rigorous analysis of printed circuit elements or printed antennas in planar multilayer media [1]-[5]. In general, the structures of interest are open geometries; hence, an integral equation (IE) is usually set up and CFGF is used as the kernel of this IE. The IE is solved using method of moments (MoM) based algorithms. Unfortunately, the cylindrical counterpart of the outlined procedure is rare because of the limitations on the available CFGF representations for cylindrically stratified media.

Manuscript received October 15, 2007; revised August 29, 2008. Current version published April 08, 2009. This work was supported in part by the Turkish Scientific and Technological Research Council under Grant EEEAG-104E044 and in part by the Turkish Academy of Sciences (TÜBA)-GEBIP

S. Karan is with the Department of Electrical and Electronics Engineering, Bilkent University, TR-06800 Bilkent, Ankara, Turkey, and also with Aselsan Electronics Inc., Ankara, Turkey.

V. B. Ertürk and A. Altintas are with the Department of Electrical and Electronics Engineering, Bilkent University, TR-06800 Bilkent, Ankara, Turkey (e-mail: vakur@ee.bilkent.edu.tr).

Color versions of one or more of the figures in this paper are available online at http://ieeexplore.iee.org.

Digital Object Identifier 10.1109/TAP.2009.2015796
A number of studies regarding the Green's functions in cylindrically stratified media have been reported before [6]-[23]. More references on the conventional spectral domain and asymptotic Green's function representations, particularly for single-layer dielectrics deposited on a perfectly conducting cylinder, can be found in [13] and [14]. However, a vast majority of the above-mentioned Green's function representations (derived for cylindrically stratified media) are not in closed form. In addition, convergence of these expressions becomes an important issue from the accuracy and efficiency points of view for antenna and/or mutual coupling problems. On the other hand, most of the studies on the subject of CFGF for cylindrically stratified media have the CFGF expressions that are valid when the source and observation points are on different radial distances from the axis of the cylinder [15]-[19]. Therefore, these expressions are useful for radiation/scattering problems, provided that the current distribution on the radiating structure is known, but cannot be used in conjunction with an MoM-based algorithm to solve antenna input impedance and mutual coupling problems. In [20], a closed-form solution for cylindrically conformal microstrip antennas is given. However, provided closed-from expressions are for the impedance matrix elements and the elements of the voltage vector (using entire domain basis functions) rather than the Green's functions. Reference [21] has presented CFGF expressions to be used in the mixed potential integral equation (MPIE). Although, these CFGF expressions (provided in [21]) are valid when the source and the observation points are located at the same radial distance from the axis of the cylinder, the final expressions are not valid along the axial line (defined as $\rho=\rho^{\prime}$ and $\phi=\phi^{\prime}$ ) of the cylinder.

In this paper, we provide the CFGF expressions that can be used as the kernel of an electric field integral equations (EFIEs) to be used in MoM-based codes to treat antenna input impedance and mutual coupling problems. Our approach starts by expressing the conventional spectral domain Green's function representations in a different form so that possible overflow/underflow problems in the numerical calculations of special cylindrical functions such as Bessel and Hankel functions can be completely avoided. As a result, the method can handle both electrically small and large cylinders. More importantly, the axial line problem can be attacked easily. Then, the summation over the cylindrical eigenmodes $n$ is performed in the spectral domain. Large $n$ values that will appear in the orders of special functions (Hankel and Bessel functions), especially for electrically large cylinders, do not create numerical problems due to the aforementioned way of 
expressing the spectral domain Green's functions. However, acceleration techniques that are presented in [21] are implemented to further increase the efficiency of this summation. Once the summation over the cylindrical eigenmodes is performed, the Fourier integral over $k_{z}$ is taken using DCIM with the help of the generalized pencil of function (GPOF) method [24], where a modified two-level approach is used. Such a modification (compared to ones presented in [15], [16], [23]) in the implementation of GPOF is critical in order to obtain accurate results in particular along the axial line. Thus, the accuracy range (defined as the distance between the source and observation points) of the CFGF expressions proposed in this study is significantly wider than that of the previously available CFGF representations. Briefly, in addition to cases where source and observation points are located at different radial distances from the axis of the cylinder, the proposed CFGF expressions are valid for almost all possible source and field points that lie on the same radial distance (such as the air-dielectric and dielectric-dielectric interfaces). The latter region includes the situation where both the source and field points are located on the axial line $\left(\rho=\rho^{\prime}\right.$ and $\left.\phi=\phi^{\prime}\right)$ of the cylinder, and valid CFGF expressions for this situation have not been available before. Furthermore, the proposed CFGF expressions work fairly well for relatively large cylinders. Consequently, they can safely be used in conjunction with MoM-based codes to investigate all aspects of printed antennas (current distribution, input impedance, radiation, etc.) and mutual coupling problems for arrays.

In Section II, the geometry and the detailed derivation of the CFGF representations are presented, which includes the modified spectral domain expressions at $\rho=\rho^{\prime}$, how the summation over the cylindrical eigenmodes is performed, solution to the axial line problem, and the implementation of GPOF with the modified two-level approach. In Section III, numerical results are given to assess the accuracy of the method. An $e^{j \omega t}$ time dependence, with $\omega$ being the angular frequency, is assumed and suppressed throughout this paper.

\section{FORMULATION}

\section{A. Geometry}

The geometry for a multilayer cylindrically stratified media is illustrated in Fig. 1. The structure is assumed to be infinite in the $z$-direction. A perfect electric conductor (PEC) cylindrical ground plane, denoted by the subscript $j=0$, forms the innermost region with a radius $a_{0}$, and material layers, denoted by the subscripts $j=1,2, \ldots$, surround the PEC region coaxially, as shown in Fig. 1 (subscript $j=1$ denotes the substrate layer; subscript $j=2$ denotes the superstrate layer, and subscript $j=3$ denotes the air layer in this figure). Each layer has a permittivity, permeability, and radius denoted by $\epsilon_{j}$, $\mu_{j}$, and $a_{j}$, respectively. Furthermore, current modes, denoted by $P^{\prime}\left(\rho^{\prime}, \phi^{\prime}, z^{\prime}\right)$ and $P(\rho, \phi, z)$, are depicted in Fig. 1 . A tangential current source is defined at an air-dielectric (or dielectric-dielectric) interface and has a dimension of $2 z_{a}$ by $2 l_{a}$ (with $l_{a}=a_{j} \phi_{a}$ ) along the $z-$ and $\phi$-directions, respectively. On the other hand, if the current mode is normal to an interface (excitation via a probe), it is usually located inside a layer, behaves
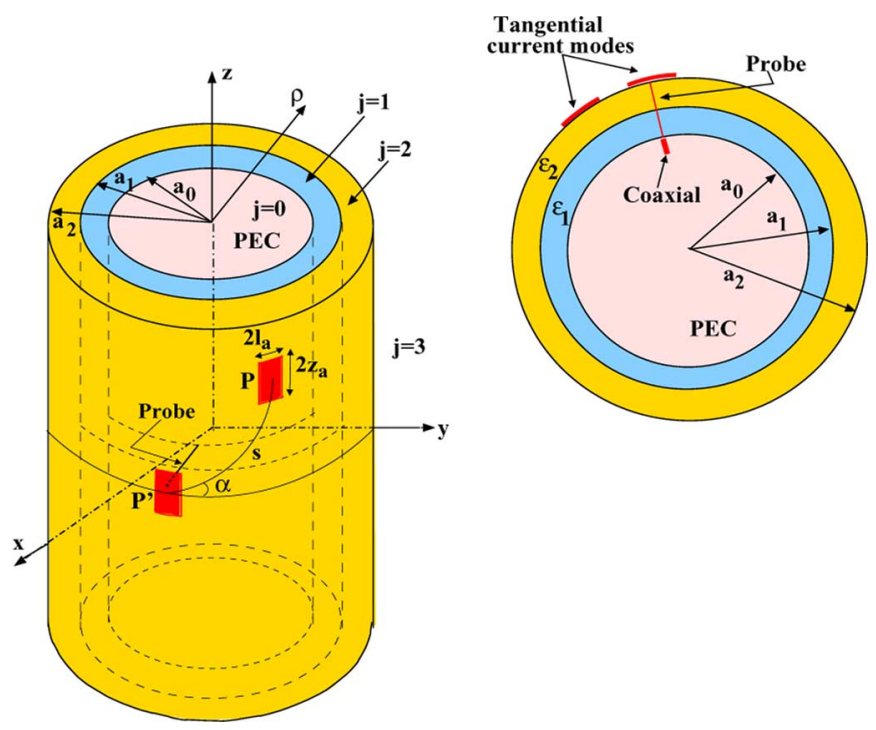

Fig. 1. Geometry of the problem. Current modes on a multilayer cylindrical structure together with cross-sectional view from the top.

like a point source in terms of $z$ and $\phi$ coordinates, and has a certain thickness along the radial (i.e., $\rho$ ) direction. Therefore, in finding the voltage vector in a MoM-based code, usually $\rho$ and $\rho^{\prime}$ are not equal to each other. The same is true for normal components of the fields due to tangential sources located at an interface. Consequently, the CFGF derivation procedure described in the following sections is not applied to current modes that are normal to interfaces. In Fig. 1, $s$ denotes the geodesic distance between the two current modes (or between the source and observation points for the CFGF expressions) and $\alpha$ is the angle between the geodesic path and the $\phi$-axis.

\section{B. Spectral Domain Green's Function Expressions When $\rho \neq \rho^{\prime}$}

In an MoM-based cylindrical microstrip antenna analysis, entries of the MoM impedance matrix require accurate representations of tangential components of the dyadic Green's function (due to tangential sources) for arbitrary source and field points that lie at the same interface. However, this is the main problem in the aforementioned CFGF expressions available in the literature. Thus, the derivation of our CFGF expressions starts with the tangential components of the spectral domain dyadic Green's function $\tilde{\overline{\mathbf{G}}}$ due to the tangential current sources. For an elementary tangential electric current source $P^{\prime}\left(\rho^{\prime}, \phi^{\prime}, z^{\prime}\right)$, the tangential components of $\tilde{\overline{\mathbf{G}}}$ for the field point $P(\rho, \phi, z)$ are given by ([16])

$$
\begin{aligned}
& \tilde{G}_{z z}=-\frac{1}{2 \omega} \sum_{n=0}^{\infty} \kappa_{n} \cos \left[n\left(\phi-\phi^{\prime}\right)\right] \frac{k_{\rho_{j}}^{2}}{\epsilon_{j}} f_{n}^{11} \\
& \frac{\tilde{G}_{\phi z}}{k_{z}}=-\frac{j}{2 \omega} \sum_{n=1}^{\infty} \sin \left[n\left(\phi-\phi^{\prime}\right)\right]\left[\frac{n}{\epsilon_{j} \rho} f_{n}^{11}+\frac{k_{\rho_{j}} j \omega \mu_{j}}{\epsilon_{j} k_{z}} \frac{\partial f_{n}^{21}}{\partial\left(k_{\rho_{j}} \rho\right)}\right] \\
& \frac{\tilde{G}_{z \phi}}{k_{z}}=-\frac{j}{2 \omega} \sum_{n=1}^{\infty} \sin \left[n\left(\phi-\phi^{\prime}\right)\right]\left[\frac{n}{\epsilon_{j} \rho^{\prime}} f_{n}^{11}-\frac{j \omega k_{\rho_{j}}}{k_{z}} \frac{\partial f_{n}^{12}}{\partial\left(k_{\rho_{j}} \rho^{\prime}\right)}\right]
\end{aligned}
$$




$$
\begin{aligned}
\tilde{G}_{\phi \phi}= & -\frac{1}{2 \omega} \sum_{n=0}^{\infty} \kappa_{n} \cos \left[n\left(\phi-\phi^{\prime}\right)\right] \\
& {\left[\frac{n^{2} k_{z}^{2}}{\epsilon_{j} \rho \rho^{\prime} k_{\rho_{j}}^{2}} f_{n}^{11}+\frac{j \omega \mu_{j} n k_{z}}{k_{\rho_{j}} \epsilon_{j} \rho^{\prime}} \frac{\partial f_{n}^{21}}{\partial\left(k_{\rho_{j}} \rho\right)}-\frac{j \omega n k_{z}}{k_{\rho_{j}} \rho} \frac{\partial f_{n}^{12}}{\partial\left(k_{\rho_{j}} \rho^{\prime}\right)}\right.} \\
& \left.+\omega^{2} \mu_{j} \frac{\partial^{2} f_{n}^{22}}{\partial\left(k_{\rho_{j}} \rho^{\prime}\right) \partial\left(k_{\rho_{j}} \rho\right)}\right]
\end{aligned}
$$

where $\kappa_{n}=0.5$ for $n=0$ and 1 , otherwise. However, since the $\rho$ related components might be important for applications involving an excitation via a probe, for the sake of completeness, these components are also given by [15]

$$
\begin{aligned}
\frac{\tilde{G}_{\rho z}}{k_{z}}= & -\frac{1}{2 \omega} \sum_{n=0}^{\infty} \kappa_{n} \cos \left[n\left(\phi-\phi^{\prime}\right)\right] \\
& \times\left[-\frac{j k_{\rho_{j}}}{\epsilon_{j}} \frac{\partial f_{n}^{11}}{\partial\left(k_{\rho_{j}} \rho\right)}+\frac{n \omega \mu_{j}}{k_{z} \epsilon_{j} \rho} f_{n}^{21}\right] \\
\tilde{G}_{\rho \phi}= & -\frac{j}{2 \omega} \sum_{n=1}^{\infty} \sin \left[n\left(\phi-\phi^{\prime}\right)\right] \\
\times & {\left[\frac{-j k_{z}}{k_{\rho_{j}}}\left(\frac{n k_{z}}{\epsilon_{j} \rho^{\prime}} \frac{\partial f_{n}^{11}}{\partial\left(k_{\rho_{j}} \rho\right)}-j \omega k_{\rho_{j}} \frac{\partial^{2} f_{n}^{12}}{\partial\left(k_{\rho_{j}} \rho\right)\left(k_{\rho_{j}} \rho^{\prime}\right)}\right)\right.} \\
& \left.\quad+\frac{n \omega \mu_{j}}{k_{\rho_{j}}^{2} \rho}\left(\frac{n k_{z}}{\epsilon_{j} \rho^{\prime}} f_{n}^{21}-j \omega k_{\rho_{j}} \frac{\partial f_{n}^{22}}{\partial\left(k_{\rho_{j}} \rho^{\prime}\right)}\right)\right]
\end{aligned}
$$$$
\frac{\tilde{G}_{z \rho}}{k_{z}}=\frac{1}{2 \omega} \sum_{n=0}^{\infty} \kappa_{n} \cos \left[n\left(\phi-\phi^{\prime}\right)\right]\left[\frac{n \omega}{k_{z} \rho^{\prime}} f_{n}^{12}+\frac{j k_{\rho_{j}}}{\epsilon_{j}} \frac{\partial f_{n}^{11}}{\partial\left(k_{\rho_{j}} \rho^{\prime}\right)}\right]
$$$$
\tilde{G}_{\phi \rho}=-\frac{j}{2 \omega} \sum_{n=1}^{\infty} \sin \left[n\left(\phi-\phi^{\prime}\right)\right]
$$$$
\times\left[\frac{n k_{z}}{k_{\rho_{j}}^{2} \rho}\left(\frac{j k_{z} k_{\rho_{j}}}{\epsilon_{j}} \frac{\partial f_{n}^{11}}{\partial\left(k_{\rho_{j}} \rho^{\prime}\right)}+\frac{n \omega}{\rho^{\prime}} f_{n}^{12}\right)\right.
$$$$
\left.+\frac{j \omega \mu_{j}}{k_{\rho_{j}}}\left(\frac{j k_{z} k_{\rho_{j}}}{\epsilon_{j}} \frac{\partial^{2} f_{n}^{21}}{\partial\left(k_{\rho_{j}} \rho\right)\left(k_{\rho_{j}} \rho^{\prime}\right)}+\frac{n \omega}{\rho^{\prime}} \frac{\partial f_{n}^{22}}{\partial\left(k_{\rho_{j}} \rho\right)}\right)\right] \text {. }
$$

However, as explained in Section II-A, $\rho$ and $\rho^{\prime}$ are not equal to each other in (5)-(8). Therefore, although the CFGF expressions due to these components [i.e., (5)-(8)] are found using the modified two-level GPOF method from the efficiency point of view, the CFGF derivation procedure, described in the following sections for (1)-(4), is not applied to $\rho$ related components. In addition, the methodology presented in [16] can also handle them [i.e., (5)-(8)].

In (1)-(8), $f_{n}^{11}, f_{n}^{12}, f_{n}^{21}$, and $f_{n}^{22}$ are the entries of $\overline{\mathbf{F}}_{n}$ (superscripts indicate entries), which is a $2 \times 2$ matrix given by

$$
\begin{aligned}
& \overline{\mathbf{F}}_{n}=H_{n}^{(2)}\left(k_{\rho_{j}} \rho\right) J_{n}\left(k_{\rho_{j}} \rho^{\prime}\right)\left[\overline{\mathbf{I}}+\frac{J_{n}\left(k_{\rho_{j}} \rho\right)}{H_{n}^{(2)}\left(k_{\rho_{j}} \rho\right)} \tilde{\overline{\mathbf{R}}}_{j, j+1}\right] \\
& \tilde{\overline{\mathbf{M}}}_{j+}\left[\overline{\mathbf{I}}+\frac{H_{n}^{(2)}\left(k_{\rho_{j}} \rho^{\prime}\right)}{J_{n}\left(k_{\rho_{j}} \rho^{\prime}\right)} \tilde{\overline{\mathbf{R}}}_{j, j-1}\right]
\end{aligned}
$$

where $k_{\rho_{j}}=\sqrt{k_{j}^{2}-k_{z}^{2}}$, with $k_{j}$ being the wave number of the medium $\left(k_{j}=\sqrt{\epsilon_{r j}} k_{0}\right)$. The expression $\tilde{\overline{\mathbf{M}}}_{j+}$ in (9) contains the $2 \times 2$ generalized reflection and transmission matrices $\tilde{\overline{\mathbf{R}}}$ and $\tilde{\overline{\mathbf{T}}}$, respectively, all of which are explicitly given in [12] and [16]. $\overline{\mathbf{I}}$ is the $2 \times 2$ identity matrix, and components that are odd functions of $k_{z}$ are divided to $k_{z}$ to make the final expression an even function of $k_{z}$.

On the other hand, note that when $\overline{\mathbf{F}}_{n}$, given by (9), is used in (1)-(8), these Green's function components become valid for $\rho>\rho^{\prime}$, and both source and field points are in the same layer. Alternative expression for $\overline{\mathbf{F}}_{n}$ that can replace (9) for $\rho^{\prime}>\rho$ can be found in [15] and [16]. However, obviously, when $\rho \rightarrow \rho^{\prime}$, $\rho^{\prime}>\rho$ and $\rho>\rho^{\prime}$ cases are equal and constitute the $\rho=\rho^{\prime}$ problem, which is the main subject of this paper.

\section{Spectral Domain Green's Function Expressions at $\left(\rho=\rho^{\prime}\right)$}

The Green's function expressions given by (1)-(4) together with (9) yield accurate results only when the source and field points are at different radial distances from the axis of the cylinder (i.e., $\rho \neq \rho^{\prime}$ ) (the same is true for the $\rho^{\prime}>\rho$ expressions). Thus, in this section, we provide expressions valid when $\rho=\rho^{\prime}$. Note that in the provided expressions, $\rho$ and $\rho^{\prime}$ are kept distinct to avoid possible confusion in explaining the methodology, in particular when handling the derivatives with respect to $\rho$ and $\rho^{\prime}$, separately.

As the first step, the spectral domain Green's function components $\tilde{G}_{u v}(u=z$ or $\phi, v=z$ or $\phi)$, given by (1)-(4) are modified for $\rho=\rho^{\prime}$ case and rewritten in the following form:

$$
\begin{array}{r}
\frac{\tilde{G}_{u v}}{k_{z}^{l}}=-\frac{1}{4 \omega} \sum_{n=-\infty}^{\infty}\left[\left(k_{\rho_{j}}^{2}\right)^{q} n^{p} H_{n}^{(2)}\left(k_{\rho_{j}} \rho\right) J_{n}\left(k_{\rho_{j}} \rho^{\prime}\right)\right. \\
\left.\times f_{u v}\left(n, k_{z}\right) e^{j n(\Delta \phi)}\right]
\end{array}
$$

where $\Delta \phi=\phi-\phi^{\prime}$, and for $u v=z z: p=0, q=1, l=0$, for $u v=\phi z: p=1, q=0, l=1$, for $u v=z \phi: p=1, q=0, l=$ 1 , and for $u v=\phi \phi: p=2, q=0, l=0$. The key term in (10) is $f_{u v}\left(n, k_{z}\right)$, explicitly given by

$$
\begin{aligned}
f_{z z}\left(n, k_{z}\right)= & \frac{f_{r 1}^{11}}{\epsilon_{j}} \\
f_{\phi z}\left(n, k_{z}\right)= & \frac{1}{\epsilon_{j} \rho} f_{r 1}^{11}+\frac{j \omega \mu_{j} k_{\rho_{j}}}{\epsilon_{j} k_{z}} f_{r 2}^{21} \\
f_{z \phi}\left(n, k_{z}\right)= & \frac{1}{\epsilon_{j} \rho^{\prime}} f_{r 1}^{11}-\frac{j \omega k_{\rho_{j}}}{k_{z}} f_{r 2}^{12} \\
f_{\phi \phi}\left(n, k_{z}\right)= & \frac{k_{z}^{2}}{\epsilon_{j} \rho \rho^{\prime} k_{\rho_{j}}^{2}} f_{r 1}^{11}+\frac{j \omega \mu_{j} k_{z}}{k_{\rho_{j}} \epsilon_{j} \rho^{\prime}} f_{r 2}^{21} \\
& -\frac{j \omega k_{z}}{k_{\rho_{j}} \rho} f_{r 3}^{12}+\omega^{2} \mu_{j} f_{r 4}^{22}
\end{aligned}
$$

where $f_{r 1}^{11}, f_{r 2}^{21}, f_{r 2}^{12}, f_{r 3}^{12}, f_{r 4}^{22}$ are the corresponding entries (each superscript indicates the corresponding entry) of $\overline{\mathbf{F}}_{r 1}, \overline{\mathbf{F}}_{r 2}$, $\overline{\mathbf{F}}_{r 3}$, and $\overline{\mathbf{F}}_{r 4}$ linked to $\overline{\mathbf{F}}_{n}$ as

$$
\begin{aligned}
\overline{\mathbf{F}}_{r 1} & =\frac{1}{H_{n}^{(2)}\left(k_{\rho_{j}} \rho\right) J_{n}\left(k_{\rho_{j}} \rho^{\prime}\right)} \overline{\mathbf{F}}_{n} \\
\overline{\mathbf{F}}_{r 2} & =\frac{1}{n H_{n}^{(2)}\left(k_{\rho_{j}} \rho\right) J_{n}\left(k_{\rho_{j}} \rho^{\prime}\right)} \frac{\partial \overline{\mathbf{F}}_{n}}{\partial\left(k_{\rho_{j}} \rho\right)}
\end{aligned}
$$




$$
\begin{aligned}
\overline{\mathbf{F}}_{r 3} & =\frac{1}{n H_{n}^{(2)}\left(k_{\rho_{j}} \rho\right) J_{n}\left(k_{\rho_{j}} \rho^{\prime}\right)} \frac{\partial \overline{\mathbf{F}}_{n}}{\partial\left(k_{\rho_{j}} \rho^{\prime}\right)} \\
\overline{\mathbf{F}}_{r 4} & =\frac{1}{n^{2} H_{n}^{(2)}\left(k_{\rho_{j}} \rho\right) J_{n}\left(k_{\rho_{j}} \rho^{\prime}\right)} \frac{\partial^{2} \overline{\mathbf{F}}_{n}}{\partial\left(k_{\rho_{j}} \rho^{\prime}\right) \partial\left(k_{\rho_{j}} \rho\right)}
\end{aligned}
$$

More explicit expressions for $\overline{\mathbf{F}}_{r 1}, \overline{\mathbf{F}}_{r 2}, \overline{\mathbf{F}}_{r 3}$, and $\overline{\mathbf{F}}_{r 4}$ are given in Appendix A [see (A.1)-(A.4)].

In (10) [together with (A.1)-(A.4)], all special cylindrical functions are expressed in the form of ratios. This procedure starts by modifying generalized reflection coefficient matrix as

$$
\begin{aligned}
\tilde{\overline{\mathbf{R}}}_{j, j-1} & =\frac{J_{n}\left(k_{\rho_{j}} a_{j-1}\right)}{H_{n}^{(2)}\left(k_{\rho_{j}} a_{j-1}\right)} \tilde{\overline{\mathbf{R}}} r_{j, j-1} \\
\tilde{\overline{\mathbf{R}}}_{j, j+1} & =\frac{H_{n}^{(2)}\left(k_{\rho_{j}} a_{j}\right)}{J_{n}\left(k_{\rho_{j}} a_{j}\right)} \tilde{\overline{\mathbf{R}}} r_{j, j+1}
\end{aligned}
$$

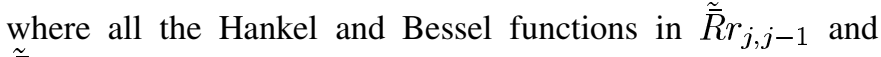
$\tilde{\bar{R}} r_{j, j+1}$ are in the form of ratios. A similar modification is performed for $\tilde{\overline{\mathbf{T}}}$ and, consequently, for $\tilde{\overline{\mathbf{M}}}_{j+}$. Furthermore, for large $n$ values, Debye representations [25] of the ratios are found in closed form and used during the summation over $n$. As a result of this step, we have achieved the following.

i) The accuracy of the summation over $n$, which exhibits convergence problems when $\rho=\rho^{\prime}$ particularly for relatively large cylinders, is improved since possible numerical overflow/underflow problems for large $n$ values are avoided.

ii) Since the ratios that use Debye representations are in closed form, the efficiency of the summation is also improved, in particular for large cylinders, as they require more terms to be summed.

iii) The form of (10) is very suitable to attack the axial line problem, which will be addressed in the following section.

To further improve the accuracy and efficiency of the summation over $n$, an envelope extraction method with respect to $n$ is applied to (10). Briefly, the limiting value of $f_{u v}\left(n, k_{z}\right)$ for very large $n$ values is numerically determined as

$$
\lim _{n \rightarrow \infty} f_{u v}\left(n, k_{z}\right) \approx C_{u v}\left(k_{z}\right)
$$

which is actually constant with respect to $n$. Then, recognizing the series expansion of $H_{0}^{(2)}\left(k_{\rho_{j}}\left|\bar{\rho}-\bar{\rho}^{\prime}\right|\right)$, given by

$$
\sum_{n=-\infty}^{\infty} H_{n}^{(2)}\left(k_{\rho_{j}} \rho\right) J_{n}\left(k_{\rho_{j}} \rho^{\prime}\right) e^{j n \Delta \phi}=H_{0}^{2}\left(k_{\rho_{j}}\left|\bar{\rho}-\bar{\rho}^{\prime}\right|\right)
$$

$C_{u v}\left(k_{z}\right)$ is subtracted from (10) and added as a function of $H_{0}^{(2)}\left(k_{\rho_{j}}\left|\bar{\rho}-\bar{\rho}^{\prime}\right|\right)$ (i.e., $\left.F_{1}^{u v}[\cdot]\right)$ with the aid of (22). In (22), $\bar{\rho}=\hat{\rho} \rho$ and $\bar{\rho}^{\prime}=\hat{\rho}^{\prime} \rho^{\prime}$, where $\hat{\rho}^{\prime}$ and $\hat{\rho}$ are unit vectors in cylindrical coordinates defined from the central axis of the cylinder

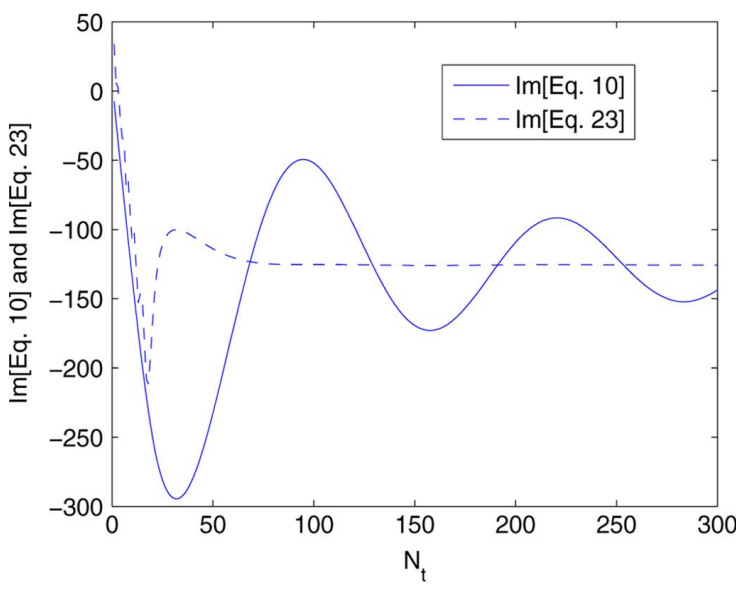

Fig. 2. Imaginary part of $\tilde{G}_{z z}$ versus $N_{t}$ using (10) and (23) for $\Delta \phi=0.05$ and $k_{z}=0$. The cylinder parameters are $a_{0}=3 \lambda_{0}, a_{1}=3.06 \lambda_{0}$, and $\epsilon_{r}=3.25$.

in the direction of source and field points, respectively. As a result, (10) becomes

$$
\begin{aligned}
\frac{\tilde{G}_{u v}}{k_{z}^{l}}= & -\frac{1}{4 \omega}\left\{\sum_{n=-\infty}^{\infty}\left(k_{\rho_{j}}^{2}\right)^{q} n^{p} H_{n}^{(2)}\left(k_{\rho_{j}} \rho\right) J_{n}\left(k_{\rho_{j}} \rho^{\prime}\right)\right. \\
& \times\left[f_{u v}\left(n, k_{z}\right)-C_{u v}\left(k_{z}\right)\right] e^{j n \Delta \phi} \\
& \left.+C_{u v}\left(k_{z}\right)\left(k_{\rho_{j}}^{2}\right)^{q} F_{1}^{u v}\left[H_{0}^{(2)}\left(k_{\rho_{j}}\left|\bar{\rho}-\bar{\rho}^{\prime}\right|\right)\right]\right\}
\end{aligned}
$$

with

$$
\begin{aligned}
& F_{1}^{z z}\left[H_{0}^{2}\left(k_{\rho_{j}}\left|\bar{\rho}-\bar{\rho}^{\prime}\right|\right)\right]=H_{0}^{2}\left(k_{\rho_{j}}\left|\bar{\rho}-\bar{\rho}^{\prime}\right|\right) \\
& F_{1}^{\phi z}\left[H_{0}^{2}\left(k_{\rho_{j}}\left|\bar{\rho}-\bar{\rho}^{\prime}\right|\right)\right]=F_{1}^{z \phi}\left[H_{0}^{2}\left(k_{\rho_{j}}\left|\bar{\rho}-\bar{\rho}^{\prime}\right|\right)\right] \\
& \quad=-j \frac{\partial H_{0}^{2}\left(k_{\rho_{j}}\left|\bar{\rho}-\bar{\rho}^{\prime}\right|\right)}{\partial \phi} \\
& \quad=-j \rho \rho^{\prime} k_{\rho_{j}} \sin \left(\phi-\phi^{\prime}\right) \frac{H_{0}^{\prime(2)}\left(k_{\rho_{j}}\left|\bar{\rho}-\bar{\rho}^{\prime}\right|\right)}{\left|\bar{\rho}-\bar{\rho}^{\prime}\right|} \\
& F_{1}^{\phi \phi}\left[H_{0}^{2}\left(k_{\rho_{j}}\left|\bar{\rho}-\bar{\rho}^{\prime}\right|\right)\right]=\frac{\partial^{2} H_{0}^{2}\left(k_{\rho_{j}}\left|\bar{\rho}-\bar{\rho}^{\prime}\right|\right)}{\partial \phi \partial \phi^{\prime}} .
\end{aligned}
$$

After taking the derivatives in (26), its right-hand side can be written explicitly as

$$
\begin{aligned}
F_{1}^{\phi \phi}[ & \left.H_{0}^{2}\left(k_{\rho_{j}}\left|\bar{\rho}-\bar{\rho}^{\prime}\right|\right)\right] \\
= & -\frac{\rho^{\prime} \rho}{\left|\bar{\rho}-\bar{\rho}^{\prime}\right|} \cos \left(\phi-\phi^{\prime}\right) k_{\rho_{j}} H_{0}^{\prime}(2)\left(k_{\rho_{j}}\left|\bar{\rho}-\bar{\rho}^{\prime}\right|\right) \\
& \quad-\frac{\rho^{\prime 2} \rho^{2}}{\left|\bar{\rho}-\bar{\rho}^{\prime}\right|^{2}} \sin ^{2}\left(\phi-\phi^{\prime}\right) k_{\rho_{j}}^{2} H_{0}^{\prime \prime}(2)\left(k_{\rho_{j}}\left|\bar{\rho}-\bar{\rho}^{\prime}\right|\right) \\
& +\frac{\rho^{\prime 2} \rho^{2}}{\left|\bar{\rho}-\bar{\rho}^{\prime}\right|^{3}} \sin ^{2}\left(\phi-\phi^{\prime}\right) k_{\rho_{j}} H_{0}^{\prime(2)}\left(k_{\rho_{j}}\left|\bar{\rho}-\bar{\rho}^{\prime}\right|\right)
\end{aligned}
$$

where ' and " denote the first and second derivatives, respectively, with respect to the argument of $H_{0}^{(2)}\left(k_{\rho_{j}}\left|\bar{\rho}-\bar{\rho}^{\prime}\right|\right)$. As a result of this step, the modified summation given by (23) converges very rapidly; hence, the limits of the infinite summation can be truncated at relatively small values $N_{t}$ (i.e., $\sum_{n=-\infty}^{\infty} \rightarrow$ $\sum_{n=-N_{t}}^{N_{t}}$ ) even for relatively large cylinders. This is illustrated in Fig. 2, where the imaginary part of $\tilde{G}_{z z}$ versus $N_{t}$ is plotted 
for $\Delta \phi=0.05$ and $k_{z}=0$ using (10) and (23) (real parts of both summations converge rapidly) for a dielectric coated PEC cylinder with $a_{0}=3 \lambda_{0}, a_{1}=3.06 \lambda_{0}\left(\lambda_{0}=\right.$ free-space wavelength), $\epsilon_{r}=3.25$.

Because the spatial domain Green's function $G_{u v}$ is related to the spectral domain Green's function $\tilde{G}_{u v}$ by an inverse Fourier transform (IFT) over $k_{z}$, given by

$$
G_{u v}=\frac{1}{2 \pi} \int_{-\infty}^{\infty} \tilde{G}_{u v} e^{-j k_{z}\left(z-z^{\prime}\right)} d k_{z}
$$

the final spectral domain expression (23) should not pose any problems for integration variable $k_{z}$. However, the following three problems have to be resolved.

i) Branch-point and pole singularities. The remedy for this is deforming the integration path as shown in Fig. 3 [15], [16]. Details of this path deformation will be given later.

ii) For small $\Delta \phi$ values (but not necessarily $\Delta \phi=0$ ), the integrand of the $k_{z}$ integral converges for very large $k_{z}$ values. Unfortunately, for large values of $k_{z}$, the imaginary part of (23) poses numerical problems (i.e., it becomes oscillatory and large). This is mainly due to the second term of (23) (i.e., due to $\left.C_{u v}\left(k_{z}\right)\left(k_{\rho_{j}}^{2}\right)^{q} F_{1}^{u v}\left[H_{0}^{(2)}\left(k_{\rho_{j}}\left|\bar{\rho}-\bar{\rho}^{\prime}\right|\right)\right]\right)$. The remedy for this problem is performing a second envelope extraction with respect to $k_{z}$, as explained below.

iii) The axial line problem, which manifests itself for all $k_{z}$ values. It is related with the argument of $H_{0}^{(2)}\left(k_{\rho_{j}}\left|\bar{\rho}-\bar{\rho}^{\prime}\right|\right)$ such that along the axial line (i.e., when $\rho=\rho^{\prime}$ and $\phi=$ $\left.\phi^{\prime}\right) H_{0}^{(2)}\left(k_{\rho_{j}}\left|\bar{\rho}-\bar{\rho}^{\prime}\right|\right)$ becomes singular. This singularity is $k_{z}$ independent and must be treated properly.

The treatment of the axial line problem is explained in the next subsection after item ii) is resolved. Therefore, as the next step to resolve item ii), another envelope extraction method with respect to $k_{z}$ (similar to the one performed in [21]) is applied to (23). Briefly, for asymptotically large $k_{z}$ value (i.e., $k_{z} \rightarrow \infty$ denoted as $k_{z \infty}$ ) on the deformed integration path $\Gamma_{3}$ in Fig. 3, the value of $C_{u v}\left(k_{z}\right)$, represented by $C_{u v}\left(k_{z \infty}\right)$, is found. Then, the product $-(1 / 4 \omega) C_{u v}\left(k_{z \infty}\right)\left(k_{\rho_{j}}^{2}\right)^{q} F_{1}^{u v}\left[H_{0}^{(2)}\left(k_{\rho_{j}}\left|\bar{\rho}-\bar{\rho}^{\prime}\right|\right)\right]$ is subtracted in the spectral domain from (23) and its Fourier transform is added to the final spatial domain Green's function representation as a function of $\left(e^{-j k_{j}\left|\bar{r}-\bar{r}^{\prime}\right|} /\left|\bar{r}-\bar{r}^{\prime}\right|\right)$ using the relation

$$
\begin{aligned}
I_{1} & =\frac{e^{-j k_{j}\left|\bar{r}-\bar{r}^{\prime}\right|}}{\left|\bar{r}-\bar{r}^{\prime}\right|} \\
& =\frac{-j}{2} \int_{-\infty}^{\infty} H_{0}^{2}\left(k_{\rho_{j}}\left|\bar{\rho}-\bar{\rho}^{\prime}\right|\right) e^{-j k_{z}\left(z-z^{\prime}\right)} d k_{z} .
\end{aligned}
$$

Consequently, the resultant expression for the spatial domain Green's function becomes

$$
\begin{aligned}
G_{u v}= & \left(\frac{j \partial}{\partial z}\right)^{l}\left\{\frac { 1 } { 2 \pi } \int _ { - \infty } ^ { \infty } \left(\frac{\tilde{G}_{u v}}{k_{z}^{l}}+\frac{1}{4 \omega} C_{u v}\left(k_{z \infty}\right)\left(k_{\rho_{j}}^{2}\right)^{q}\right.\right. \\
& \left.F_{1}^{u v}\left[H_{0}^{(2)}\left(k_{\rho_{j}}\left|\bar{\rho}-\bar{\rho}^{\prime}\right|\right)\right]\right) e^{-j k_{z}\left(z-z^{\prime}\right)} d k_{z} \\
& \left.-\frac{j}{4 \pi \omega} C_{u v}\left(k_{z \infty}\right)\left(k_{j}^{2}+\frac{\partial^{2}}{\partial z^{2}}\right)^{q} F_{2}^{u v}\left[I_{1}\right]\right\}
\end{aligned}
$$

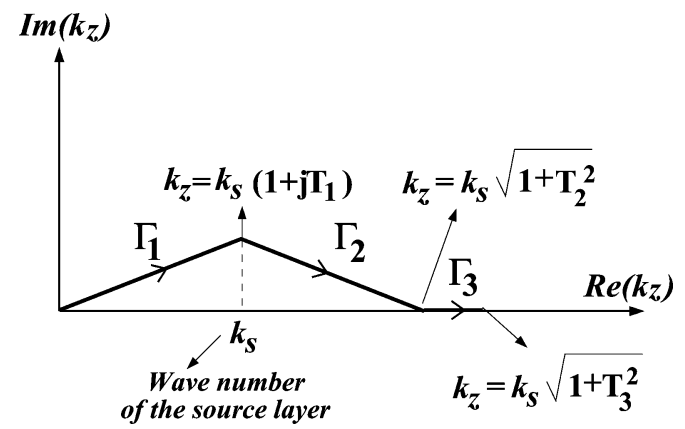

Fig. 3. Deformed integration path.

where

$$
\begin{aligned}
F_{2}^{z z}\left[I_{1}\right] & =\frac{e^{-j k_{j}\left|\bar{r}-\bar{r}^{\prime}\right|}}{\left|\bar{r}-\bar{r}^{\prime}\right|} \\
F_{2}^{\phi z}\left[I_{1}\right] & =F_{2}^{z \phi}\left[I_{1}\right]=-j \frac{\rho^{\prime} \rho}{\left|\bar{\rho}-\bar{\rho}^{\prime}\right|} \sin \left(\phi-\phi^{\prime}\right) \frac{\partial I_{1}}{\partial\left|\bar{\rho}-\bar{\rho}^{\prime}\right|} \\
& =-j \rho^{\prime} \rho \sin \left(\phi-\phi^{\prime}\right)\left[-j k_{j} \frac{e^{-j k_{j}\left|\bar{r}-\bar{r}^{\prime}\right|}}{\left|\bar{r}-\bar{r}^{\prime}\right|^{2}}-\frac{e^{-j k_{j}\left|\bar{r}-\bar{r}^{\prime}\right|}}{\left|\bar{r}-\bar{r}^{\prime}\right|^{3}}\right]
\end{aligned}
$$

$$
\begin{aligned}
F_{2}^{\phi \phi}\left[I_{1}\right]= & {\left[-\frac{\rho^{\prime} \rho}{\left|\bar{\rho}-\bar{\rho}^{\prime}\right|} \cos \left(\phi-\phi^{\prime}\right)\right.} \\
& \left.+\frac{\rho^{\prime 2} \rho^{2}}{\left|\bar{\rho}-\bar{\rho}^{\prime}\right|^{3}} \sin ^{2}\left(\phi-\phi^{\prime}\right)\right] \frac{\partial I_{1}}{\partial\left|\bar{\rho}-\bar{\rho}^{\prime}\right|} \\
& -\frac{\rho^{\prime 2} \rho^{2}}{\left|\bar{\rho}-\bar{\rho}^{\prime}\right|^{2}} \sin ^{2}\left(\phi-\phi^{\prime}\right) \frac{\partial^{2} I_{1}}{\partial\left|\bar{\rho}-\bar{\rho}^{\prime}\right|^{2}} \\
= & {\left[\rho^{\prime} \rho \cos \left(\phi-\phi^{\prime}\right)-\frac{\rho^{\prime 2} \rho^{2}}{\left|\bar{\rho}-\bar{\rho}^{\prime}\right|^{2}} \sin ^{2}\left(\phi-\phi^{\prime}\right)\right] } \\
& \times\left[j k_{j} \frac{e^{-j k_{j}\left|\bar{r}-\bar{r}^{\prime}\right|}}{\left|\bar{r}-\bar{r}^{\prime}\right|^{2}}+\frac{e^{-j k_{j}\left|\bar{r}-\bar{r}^{\prime}\right|}}{\left|\bar{r}-\bar{r}^{\prime}\right|^{3}}\right] \\
& +\rho^{\prime 2} \rho^{2} \sin ^{2}\left(\phi-\phi^{\prime}\right)\left[\frac{j k_{j}}{\left|\bar{\rho}-\bar{\rho}^{\prime}\right|^{2}} \frac{e^{-j k_{j}\left|\bar{r}-\bar{r}^{\prime}\right|}}{\left|\bar{r}-\bar{r}^{\prime}\right|^{2}}\right. \\
& +\frac{1}{\left|\bar{\rho}-\bar{\rho}^{\prime}\right|^{2}} \frac{e^{-j k_{j}\left|\bar{r}-\bar{r}^{\prime}\right|}}{\left|\bar{r}-\bar{r}^{\prime}\right|^{3}}+k_{j}^{2} \frac{e^{-j k_{j}\left|\bar{r}-\bar{r}^{\prime}\right|}}{\left|\bar{r}-\bar{r}^{\prime}\right|^{3}} \\
& \left.-3 j k_{j} \frac{e^{-j k_{j}\left|\bar{r}-\bar{r}^{\prime}\right|}}{\left|\bar{r}-\bar{r}^{\prime}\right|^{4}}-3 \frac{e^{-j k_{j}\left|\bar{r}-\bar{r}^{\prime}\right|}}{\left|\bar{r}-\bar{r}^{\prime}\right|^{5}}\right] .
\end{aligned}
$$

In arriving at (31)-(33), in addition to (29), the following two relations are used:

$$
\begin{aligned}
& \frac{-j}{2} \int_{-\infty}^{\infty} k_{\rho_{j}} H_{0}^{\prime(2)}\left(k_{\rho_{j}}\left|\bar{\rho}-\bar{\rho}^{\prime}\right|\right) e^{-j k_{z}\left(z-z^{\prime}\right)} d k_{z}=\frac{\partial I_{1}}{\partial\left|\bar{\rho}-\bar{\rho}^{\prime}\right|} \\
& \frac{-j}{2} \int_{-\infty}^{\infty} k_{\rho_{j}}^{2} H_{0}^{\prime \prime}(2)\left(k_{\rho_{j}}\left|\bar{\rho}-\bar{\rho}^{\prime}\right|\right) e^{-j k_{z}\left(z-z^{\prime}\right)} d k_{z}=\frac{\partial^{2} I_{1}}{\partial\left|\bar{\rho}-\bar{\rho}^{\prime}\right|^{2}} .
\end{aligned}
$$

Although the spatial domain Green's function representation given by (30) is not in closed form, the integrand is now fast decaying with respect to $k_{z}$ even for very small $\Delta \phi$ values except the axial line (i.e., $\Delta \phi=0$ ). This is illustrated in Fig. 4, where 


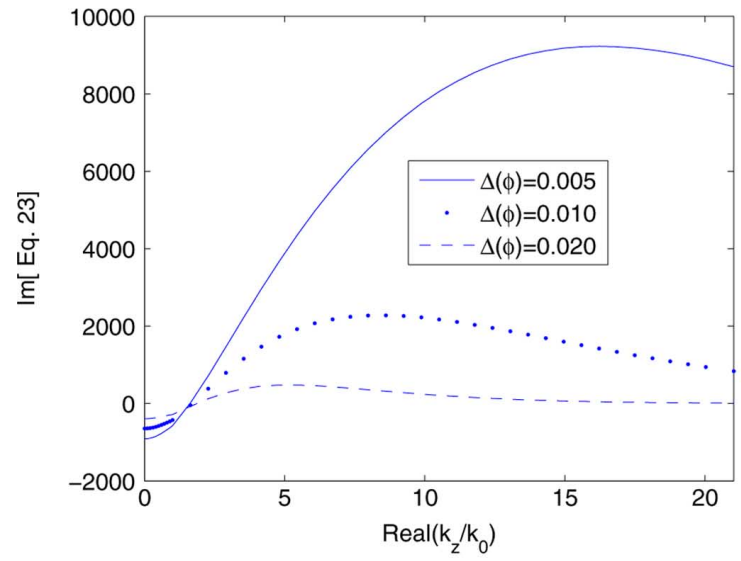

(a)

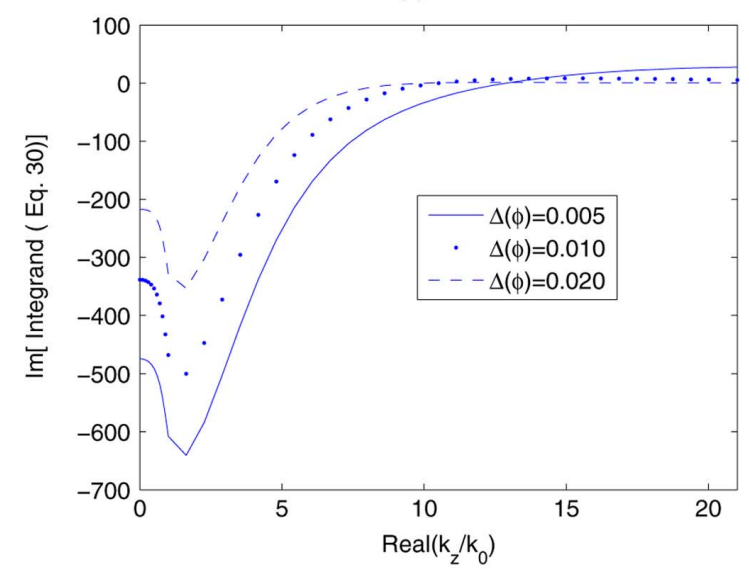

(b)

Fig. 4. The imaginary parts of (a) $\tilde{G}_{z z}$ in (23) and (b) the integrand of (30) versus real $k_{z}$ for different $\Delta \phi$ (in radians) values for the same cylinder parameters given in Fig. 2.

the imaginary part of (23) and the imaginary part of the integrand of (30) are plotted versus real $k_{z}$ for different $\Delta \phi$ values for the aforementioned cylinder $\left(a_{0}=3 \lambda_{0}, a_{1}=3.06 \lambda_{0}, \epsilon_{r}=\right.$ 3.25). As seen in Fig. 4, while (23) becomes problematic for large $k_{z}$ values [see Fig. 4(a)], especially when $\Delta \phi=0.005$, the integrand of (30) is well behaved and converges to zero as desired [see Fig. 4(b)]. Note that again, the real parts of (23) and integrand of (30) do not pose any difficulty.

\section{Solution to the Axial Line Problem}

The axial line problem manifests itself particularly for $G_{z z}$ and $G_{\phi \phi}$ components. The $H_{0}^{(2)}\left(k_{\rho_{j}}\left|\bar{\rho}-\bar{\rho}^{\prime}\right|\right)$ related terms in (30) yield singularity problems along the axial line since the argument of the Hankel function becomes zero. Although the same is true for $G_{\phi z}=G_{z \phi}$ components, the value of them along the axial line is actually zero since they possess a $\sin (2 \alpha)$ type variation ([26]), where $\alpha$ is shown in Fig. 1.

The problematic terms in the integrand of (30) for $u v=z z$ and $u v=\phi \phi$ are (24), (26), and (27), respectively, since the former one has $H_{0}^{2}\left(k_{\rho_{j}}\left|\bar{\rho}-\bar{\rho}^{\prime}\right|\right)$ and the latter one contains its derivatives with respect to both $\phi$ and $\phi^{\prime}$. Therefore, for $u v=z z$, first we use the small argument approximation of $H_{0}^{2}\left(k_{\rho_{j}}\left|\bar{\rho}-\bar{\rho}^{\prime}\right|\right)$ given by

$$
H_{0}^{2}\left(k_{\rho_{j}}\left|\bar{\rho}-\bar{\rho}^{\prime}\right|\right) \approx 1-j \frac{2}{\pi} \log \left(\frac{\gamma k_{\rho_{j}}\left|\bar{\rho}-\bar{\rho}^{\prime}\right|}{2}\right)
$$

where $\gamma=1.781$. Then, considering that we are working along the axial line, where $\rho=\rho^{\prime}, \phi \rightarrow \phi^{\prime}$ (and hence, $\sin (\Delta \phi) \approx$ $\Delta \phi)$, and making use of the properties of the log function, we rewrite the small argument approximation of $H_{0}^{2}\left(k_{\rho_{j}}\left|\bar{\rho}-\bar{\rho}^{\prime}\right|\right)$ as

$$
H_{0}^{2}\left(k_{\rho_{j}}\left|\bar{\rho}-\bar{\rho}^{\prime}\right|\right) \approx 1-j \frac{2}{\pi} \log \left(\gamma k_{\rho_{j}} \rho\right)-j \frac{2}{\pi} \log \left(\frac{\Delta \phi}{2}\right) .
$$

As seen in (37), the last term $-j(2 / \pi) \log (\Delta \phi / 2)$ is a logarithmic singularity that is responsible from the problems along the axial line and is $k_{z}$ independent. Because we are seeking to find accurate CFGF expressions to be used in conjunction with an MoM code, the logarithmic singular part converges to zero during the mutual impedance calculations [as defined in (44)] in a Galerkin-type MoM procedure due to performing the integrals over the surface areas of basis and testing current modes. As a result, with the aid of mutual impedance calculations, accurate solution along the axial line is achieved.

A similar approach is followed for the $G_{\phi \phi}$ component. However, it is noticed that starting with the right-hand side of (27) leads to a singularity, which is not integrable as opposed to the $G_{z z}$ component. Therefore, our starting point for the $G_{\phi \phi}$ component is to use (26) in the mutual impedance calculations along the axial line. Then, in a Galerkin-type MoM procedure, first selecting the basis and testing functions differentiable with respect to $\phi$ and $\phi^{\prime}$, and performing an integration by parts twice, the derivatives acting on $H_{0}^{2}\left(k_{\rho_{j}}\left|\bar{\rho}-\bar{\rho}^{\prime}\right|\right)$ are transferred to the basis and testing functions. As a result, the problematic term in the integrand of (30) for $u v=\phi \phi$ is again $H_{0}^{2}\left(k_{\rho_{j}}\left|\bar{\rho}-\bar{\rho}^{\prime}\right|\right)$, which leads to a logarithmic singularity along the axial line. Therefore, one can repeat the same steps performed for the $G_{z z}$ component and can show that in the mutual impedance calculations, the singular term will converge to zero. Consequently, an accurate solution for the $G_{\phi \phi}$ component is achieved along the axial line.

\section{E. Closed-Form Representations in the Spatial Domain}

In this section, we present how the final CFGF expressions in the spatial domain are obtained by evaluating the integral part of (30) in closed form [making use of (37) along the axial line] using the DCIM with the aid of the GPOF method. Our two-level GPOF implementation presented here partially differs from what [15], [16], and [23] have presented. Therefore, in addition to a brief discussion on how GPOF is implemented in [15], [16], and [23] with some numerical problems they may experience when $\rho=\rho^{\prime}$, this section includes some remarks on the accuracy range of the final spatial domain CFGF expressions as well as how nonspherical waves affect this accuracy range.

The first step in any GPOF implementation is to sample the spectral domain Green's functions on a path that is free from 
singularities. Considering the fact that the integrand of the integral part of (30) is an even function of $k_{z}$, the IFT integral is folded to a zero to $\infty$ integral given by

$$
\begin{aligned}
G_{u v}^{\mathrm{GPOF}}= & \frac{1}{\pi} \int_{0}^{\infty} \tilde{G}_{u v}^{\mathrm{GPOF}} \cos \left[k_{z}\left(z-z^{\prime}\right)\right] d k_{z} \\
= & \frac{1}{\pi} \int_{0}^{\infty}\left(\frac{\tilde{G}_{u v}}{k_{z}^{l}}+\frac{1}{4 \omega} C_{u v}\left(k_{z \infty}\right)\left(k_{\rho_{j}}^{2}\right)^{q}\right. \\
& \left.F_{1}^{u v}\left[H_{0}^{(2)}\left(k_{\rho_{j}}\left|\bar{\rho}-\bar{\rho}^{\prime}\right|\right)\right]\right) \cos \left[k_{z}\left(z-z^{\prime}\right)\right] d k_{z}
\end{aligned}
$$

and similar to [16], the original path is deformed as shown in Fig. 3 to overcome the effects of the pole and branch-point singularities. The parameters that define the deformed integration path are as follows. On the first path named as $\Gamma_{1}$, for $0 \leq t_{1}<T_{1}, k_{z}$ is defined as

$$
k_{z}=k_{s}\left(1+j T_{1}\right) \frac{t_{1}}{T_{1}}
$$

on the second path named as $\Gamma_{2}$, for $0 \leq t_{2}<T_{2}-T_{1}, k_{z}$ is defined as

$$
k_{z}=k_{s}\left[1+j T_{1}+\left(\sqrt{1+T_{2}^{2}}-1-j T_{1}\right) \frac{t_{2}}{T_{2}-T_{1}}\right]
$$

and on the third path named as $\Gamma_{3}$, for $0 \leq t_{3}<T_{3}-T_{2}, k_{z}$ is defined as

$$
k_{z}=k_{s}\left[\sqrt{1+T_{2}^{2}}+\left(\sqrt{1+T_{3}^{2}}-\sqrt{1+T_{2}^{2}}\right) \frac{t_{3}}{T_{3}-T_{2}}\right]
$$

where $k_{s}$ is the wave number of the source layer.

The two-level GPOF implementation in [15] and [16] is very similar to that presented in [4] and [5]. Briefly, the spectral domain Green's functions are sampled uniformly on $\Gamma_{3}$, approximated in terms of complex exponentials via GPOF, and the corresponding CFGFs in the spatial domain are found with the aid of the large argument approximation of the zeroth-order Hankel function and the Sommerfeld identity. Then, the approximated spectral domain Green's functions are subtracted from the original spectral domain Green's functions, and resulting expressions are uniformly sampled on $\Gamma_{1}$ and $\Gamma_{2}$, approximated in terms of complex exponentials via GPOF and transformed to the spatial domain in closed-forms. Addition of the two steps constitutes the final CFGF expressions in the spatial domain. However, this approach yields inaccurate results due to numerical problems when the value of the spectral domain Green's functions on $\Gamma_{3}$ is large, which is usually the case when $\rho=\rho^{\prime}$, particularly along the paraxial region (i.e., $\Delta \phi \rightarrow 0$ ) of the cylinder. On the other hand, in [23], a one-level GPOF implementation is used, which requires again taking samples and approximating the spectral domain Green's functions on $\Gamma_{3}$. Such an implementation may lead to numerical problems as well as inaccuracies when source and observation points are well separated $\left(3 \lambda_{0}-4 \lambda_{0}\right)$. As explained before, performing the envelope extraction with respect to $k_{z}$ helps us to obtain small-valued spectral domain Green's functions on $\Gamma_{3}$ but requires a modification in the approach.

Therefore, in our two-level GPOF implementation, first we notice that the spectral domain samples of $\tilde{G}_{u v}^{\mathrm{GPOF}}$ on $\Gamma_{3}$ are very small and almost constant. Denoting this term as $\tilde{G}_{u v}^{\mathrm{GPOF}_{3}}$, we subtract $\tilde{G}_{u v}^{\mathrm{GPOF}_{3}}$ from $\tilde{G}_{u v}^{\mathrm{GPOF}}$, and the resulting expression $\tilde{G}_{u v}^{\mathrm{GPOF}}-\tilde{G}_{u v}^{\mathrm{GPOF}} \mathrm{F}_{3}$ is sampled uniformly on $\Gamma_{1}$ and $\Gamma_{2}$ by taking $N_{1}$ and $N_{2}$ samples, respectively. Then, the sampled Green's functions are approximated in terms of $M_{1}$ and $M_{2}$ complex exponentials of $k_{z}$ via the GPOF method, resulting

$$
\begin{aligned}
\tilde{G}_{u v}^{k_{z}} \cong & \sum_{n=1}^{M_{1}} b_{n_{t}} e^{s_{n_{t}} t_{1}}+\sum_{m=1}^{M_{2}} b_{m_{t}} e^{s_{m_{t}} t_{2}} \\
& + \text { contribution }\left(\tilde{G}_{u v}^{\mathrm{GPOF}_{3}}\right) \\
\cong & \sum_{n=1}^{M_{1}} b_{n_{k}} e^{k_{z} s_{n_{k}}}+\sum_{m=1}^{M_{2}} b_{m_{k}} e^{k_{z} s_{m_{k}}} \\
& + \text { contribution }\left(\tilde{G}_{u v}^{\mathrm{GPOF}} \mathrm{FOF}_{3}\right) .
\end{aligned}
$$

Transforming $\tilde{G}_{u v}^{k_{z}}$ into the spatial domain via

$$
G_{u v}^{\mathrm{GPOF}}=\frac{1}{\pi} \int_{\Gamma_{1}+\Gamma_{2}} \tilde{G}_{u v}^{k_{z}} \cos \left[k_{z}\left(z-z^{\prime}\right)\right] d k_{z}
$$

and performing the integral given by (43) in closed form, $G_{u v}^{\mathrm{GPOF}}$, which is originally given by (38), is obtained in closed form. The final CFGF expressions in the spatial domain then become the addition of $G_{u v}^{\text {GPOF }}$ and the closed-form part of (30) given by $-(j / 4 \pi \omega) C_{u v}\left(k_{z \infty}\right)\left(k_{j}^{2}+\left(\partial^{2} / \partial z^{2}\right)\right)^{q} F_{2}^{u v}\left[I_{1}\right]$.

In (42), the last term, contribution $\left(\tilde{G}_{u v}^{\mathrm{GPOF}}{ }^{3}\right)$, represents the contribution coming from $\Gamma_{3}$. Unlike the previously published works ([15]-[19] and [21]-[23]), its contribution is not taken in this paper. The main reason is that after the envelope extraction with respect to $k_{z}$, the contribution coming from $\Gamma_{3}$ is not large even for relatively small $k_{z}$ values. Furthermore, by choosing the $T_{2}$ parameter for $\Gamma_{2}$ relatively large with respect to the same (or similar) parameter chosen in [15]-[19] and [21]-[23], $\tilde{G}_{u v}^{\mathrm{GPOF}}$ on $\Gamma_{3}$ becomes negligible. It should be noted that there is, however, an additional cost for this implementation: both the number of samples taken on $\Gamma_{2}$ and the corresponding number of approximating complex exponentials are increased. One can reduce the value of $T_{2}$, which in turn shortens the path $\Gamma_{2}$. In this case, although $\tilde{G}_{u v}^{\mathrm{GPOF}}$ on $\Gamma_{3}$ is not large (because of the envelope extraction with respect to $k_{z}$ ), it is not negligible. Thus, such an implementation requires one to approximate $\tilde{G}_{u v}^{\mathrm{GPOF}}$ in terms of complex exponentials on this path. The latter situation was tried but abandoned in this work because of the efficiency and accuracy problems.

Lastly, we find it useful to add a brief discussion on the accuracy range (defined as the distance between the source and observation points) and effects of nonspherical waves to this accuracy range of the final spatial domain CFGF expressions. First, because the derived CFGF expressions constitute the kernel of the EFIE, they experience a relatively severe singularity when the source and observation points overlap with each. However, at present, such a singularity (in the proposed CFGF representations) is not an integrable singularity. Hence, in an MoMbased code, alternative Green's function representations (unfortunately not available in closed-form) must be used for the entries of the MoM impedance matrix that represent the self and overlapping terms. On the other hand, it is well known that (see, for example, the explanations in [5]) in the course of ob- 


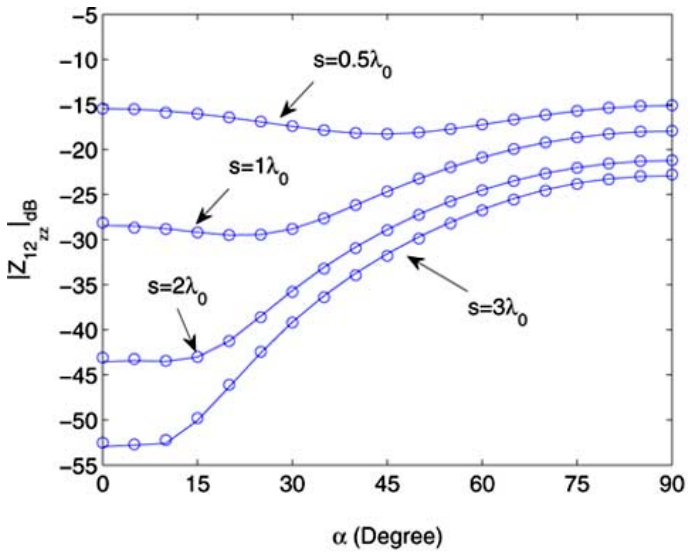

(a)

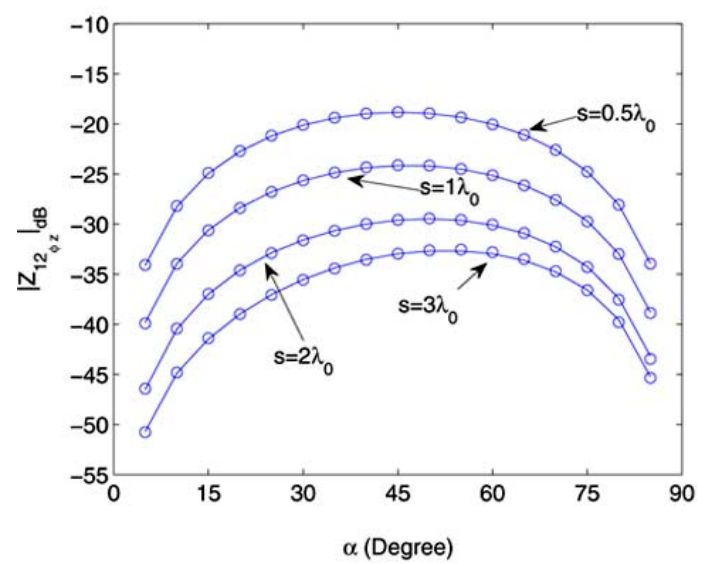

(c)

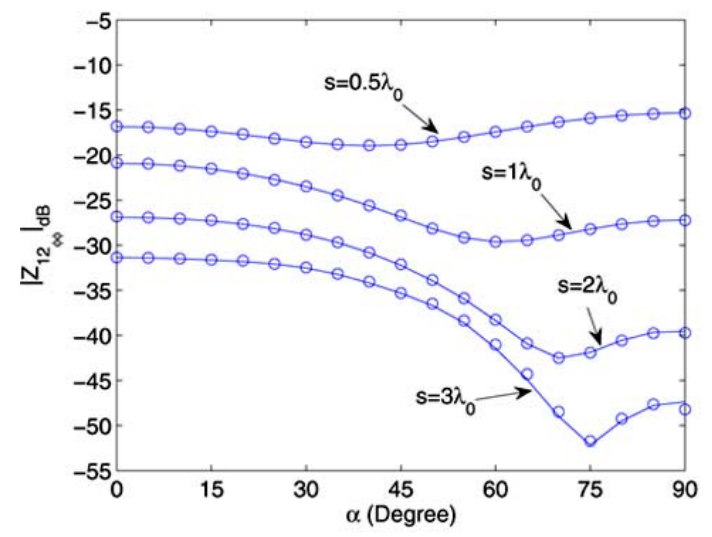

(e)

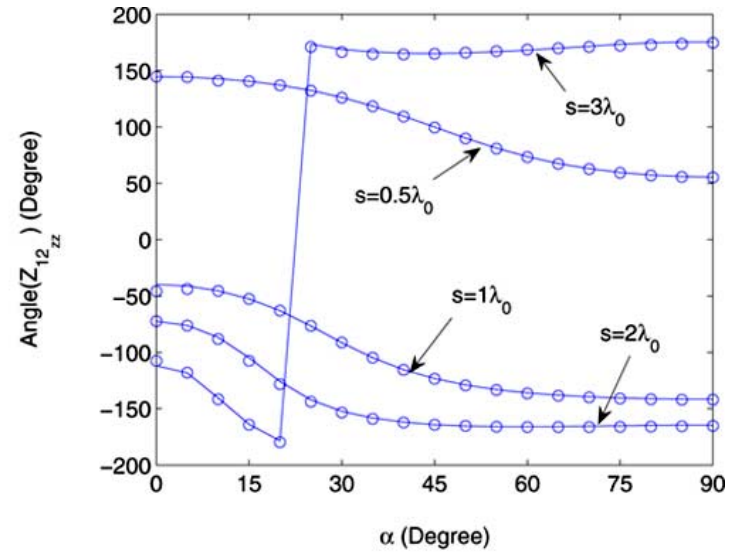

(b)

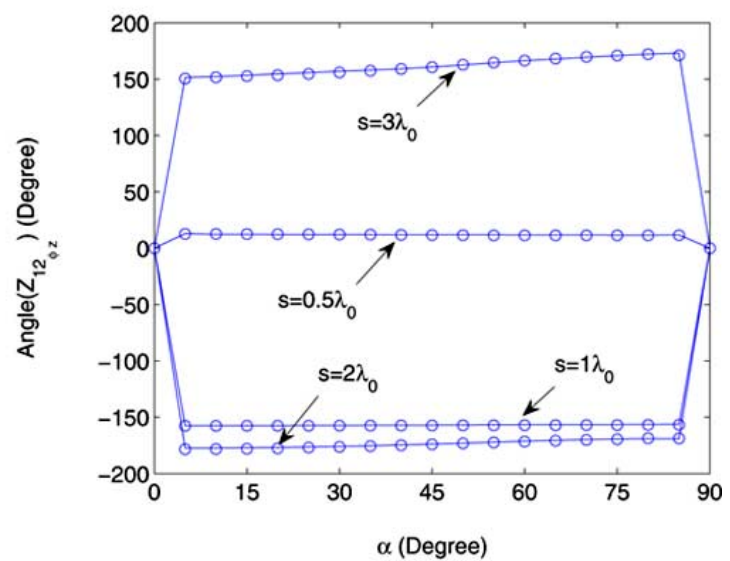

(d)

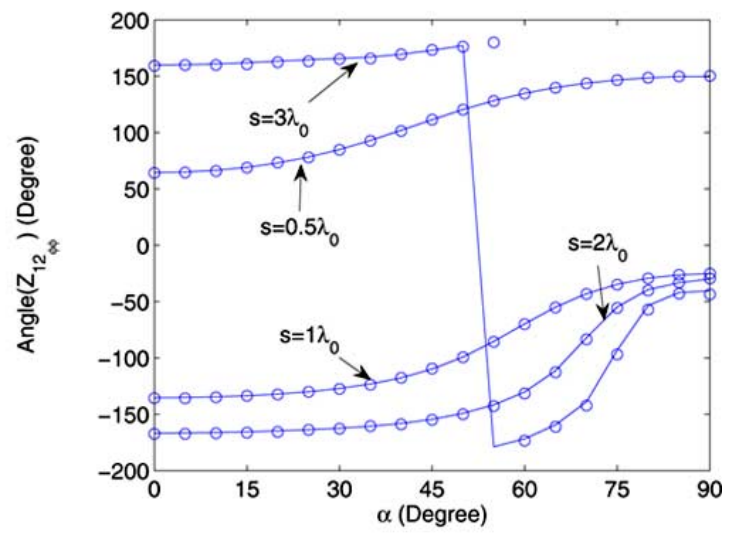

(f)

Fig. 5. Magnitude and phase of the mutual impedance $Z_{12 u v}$ between $\hat{u}$ - and $\hat{v}$-directed current sources for the coated cylinder with the parameters given in Fig. 2. (Solid line) eigenfunction solution; (circles) CFGF solution.

taining CFGF expressions, the approximating functions represent spherical waves with complex distances. Therefore, types of waves that are different in nature than the spherical waves, such as surface waves and/or lateral waves, are not properly accounted and should be treated explicitly if it is desired to accurately include their effects. Inaccurate inclusion of their effects leads to some limitations in the accuracy range of the final CFGF expressions. For planar cases, it has been shown that [3]-[5] if GPOF is implemented correctly, results are very accurate up to a few free-space wavelengths. Beyond this separation, where surface-wave contributions start to dominate, explicit treatment of surface waves might be necessary. However, treatment of surface waves does not affect the robustness of the method, and results are still in closed form. In this paper, we have not treated the surface waves explicitly and, as will be shown in the numerical results, our EFIE-related CFGFs are accurate up to $6 \lambda_{0}-7 \lambda_{0}$ (or even more for some components). Similar to the planar case, explicit treatment of surface waves can be performed. 


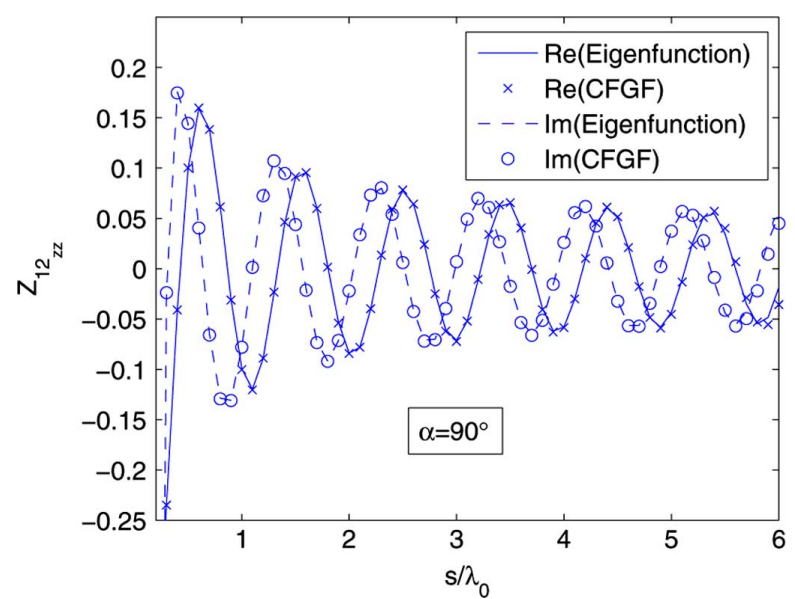

Fig. 6. Real and imaginary parts of the mutual impedance $\left(Z 12_{z z}\right)$ between two identical $\hat{z}$-directed current sources versus separation when $\alpha=90^{\circ}$ (axial line) for the coated cylinder with the parameters given in Fig. 2.

\section{NUMERICAL RESULTS}

To assess the accuracy of this method, some numerical results in the form of mutual impedance between two tangential, nontouching electric current modes $J_{1_{u}}$ and $J_{2_{v}}$ are obtained using the proposed CFGF expressions and compared with a standard eigenfunction solution in the spectral domain (used as a reference solution) for a dielectric coated circular PEC cylinder with $a_{0}=3 \lambda_{0}, a_{1}=3.06 \lambda_{0}, \epsilon_{r}=3.25$. The mutual impedance $Z_{12_{u v}}$ between the current modes is simply given by

$$
Z_{12_{u v}}=\int_{S_{2}} E_{1_{u}} J_{2_{v}} d s
$$

where $E_{1_{u}}$ is the field due to the current mode $J_{1_{u}}$ and $S_{2}$ is the area occupied by the current mode $J_{2_{v}}$. The current modes are defined by a piecewise sinusoid along the direction of the current and by a constant along the direction perpendicular to the current [26]. Each element has dimensions of $0.1 \lambda_{0}$ (along the direction of the current) by $0.04 \lambda_{0}$. This particular choice of current modes guarantees the convergence of the eigenfunction solution, even though the rate of convergence is very slow. In Fig. 5, the magnitude (in $\mathrm{dB}$ ) and phase of mutual impedances $Z_{12_{z z}}, Z_{12_{\phi z}}$, and $Z_{12_{\phi \phi}}$ versus $\alpha$ for various $s$ values are shown. The parameters are chosen the same as in [27] and [28], yielding a duplication of the results presented previously in [27] and [28]. Very good agreement is obtained between the eigenfunction solution and the new CFGF expressions.

The next set of results are presented to show the accuracy of our CFGF expressions along the axial line, which has been remaining as a problematic region in the previous studies. Therefore, in Figs. 6 and 7, the real and imaginary parts of the mutual impedance between two identical $\hat{z}$ - and $\hat{\phi}$-directed current modes versus separation for $\alpha=90^{\circ}$ are depicted, respectively. Similar to the previous numerical example, the parameters are chosen the same as in [29]. While there is a very good agreement between the eigenfunction solution and the proposed CFGF expressions along the axial line, the disagreement seen in Fig. 7 after $5 \lambda_{0}$ is due to the convergence problems of the eigenfunction solution. Note that the proposed CFGF expressions are accurate for distances more than $6-7 \lambda_{0}$ (tested with the high-fre-

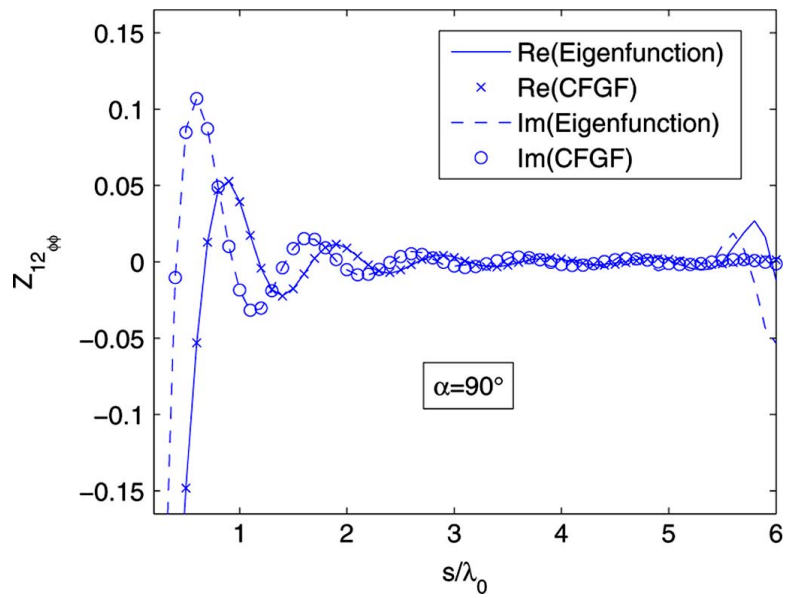

Fig. 7. Real and imaginary parts of the mutual impedance $\left(Z 12_{\phi \phi}\right)$ between two identical $\hat{\phi}$-directed current sources versus separation when $\alpha=90^{\circ}$ (axial line) for the coated cylinder with the parameters given in Fig. 2.

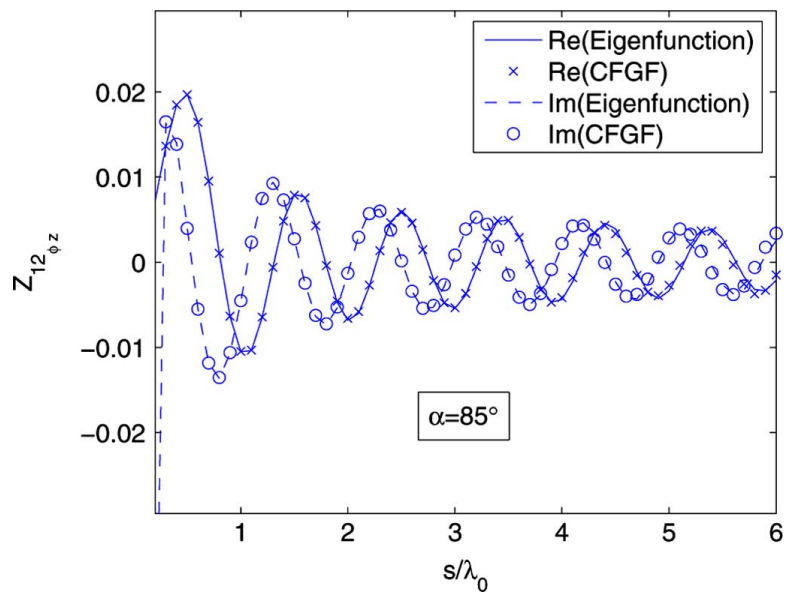

Fig. 8. Real and imaginary parts of the mutual impedance $\left(Z 12_{\phi z}\right)$ between a $\hat{\phi}$ - and a $\hat{z}$-directed current source versus separation when $\alpha=85^{\circ}$ (nearly axial line) for the coated cylinder with the parameters given in Fig. 2.

quency based asymptotic solutions). In a similar fashion, Fig. 8 illustrates the mutual impedance between a $\hat{z}$-and a $\hat{\phi}$-directed current source versus separation for $\alpha=85^{\circ}$. Keeping in mind that since this component possesses a $\sin (2 \alpha)$-type variation as seen in Fig. 5(c), the mutual impedance is zero along the axial direction. Therefore, for this particular case, the angle $\alpha$ is set to $85^{\circ}$.

Lastly, for the generation of all these CFGF results, the following parameters are used. The path, shown in Fig. 3, is formed by defining $T_{1}=0.1, T_{2}=20$ and $T_{3}=22$. On $\Gamma_{1}$ and $\Gamma_{2}$, $N_{1}=30$ and $N_{2}=250$ spectral domain samples are used, respectively. These samples are approximated in terms of $M_{1}=5$ and $M_{2}=7$ complex exponentials in the spatial domain. Furthermore, approximately $15 k_{0} a_{j}$ ( $j$ belongs to the larger radius, $k_{0}=2 \pi / \lambda_{0}$ ) terms are used for the summation over the cylindrical eigenmodes in the spectral domain. This number is actually quite larger than what is necessary for the convergence of the summation but chosen as is to leave a safety margin. However, this summation is still one of the main bottlenecks of the method. Some work is in progress to further accelerate the summation as well as to optimize the parameters $N_{1}, N_{2}, M_{1}, M_{2}$. 


\section{CONCLUSION}

CFGF expressions, which constitute the kernel of an EFIE for cylindrically stratified media, are developed. Because these CFGFs are very accurate for almost all possible source and field points, they can be used in conjunction with MoM-based codes to investigate microstrip structures such as antennas and arrays that are printed on several layers of cylindrically stratified media. Such an investigation might be radiation/scattering from a printed antenna/array on a coated cylinder, input impedance of a microstrip antenna (isolated or in the presence of other elements), or mutual coupling among various printed array elements.

Besides the fact that the validity range of the developed CFGFs is significantly improved compared to all CFGF expressions available in the literature for cylindrically stratified media, several analytical and numerical techniques presented before are implemented i) to accelerate the method and ii) to avoid possible numerical problems, in particular due to cylindrical special functions. Therefore, printed elements on electrically large cylinders that are in general analyzed with high-frequency based methods can also be handled with the proposed CFGFs. The proposed CFGFs are not valid in the region where the source region and field points overlap, and they become less accurate when distances between the source and field points are very large where only surface-wave contributions are dominant. The former one is due to the fact that kernels of EFIE are always more singular compared to other integral equations, and the latter one is due to the fact that surface waves are not represented properly.

Proper inclusion of surface wave contributions and development of alternative (approximate) CFGF representations to be used when the source region and field points overlap are currently being investigated.

\section{APPENDIX}

A. Explicit Expressions for $\overline{\mathbf{F}}_{r 1}, \overline{\mathbf{F}}_{r 2}, \overline{\mathbf{F}}_{r 3}$, and $\overline{\mathbf{F}}_{r 4}$

$$
\begin{aligned}
\overline{\mathbf{F}}_{r 1}= & {\left[\overline{\mathbf{I}}+\frac{H_{n}^{(2)}\left(k_{\rho_{j}} a_{j}\right)}{H_{n}^{(2)}\left(k_{\rho_{j}} \rho\right)} \frac{J_{n}\left(k_{\rho_{j}} \rho\right)}{J_{n}\left(k_{\rho_{j}} a_{j}\right)} \tilde{\overline{\mathbf{R}}} r_{j, j+1}\right] \tilde{\overline{\mathbf{M}}}_{j+} } \\
& \times\left[\overline{\mathbf{I}}+\frac{J_{n}\left(k_{\rho_{j}} a_{j-1}\right)}{J_{n}\left(k_{\rho_{j}} \rho^{\prime}\right)} \frac{H_{n}^{(2)}\left(k_{\rho_{j}} \rho^{\prime}\right)}{H_{n}^{(2)}\left(k_{\rho_{j}} a_{j-1}\right)} \tilde{\overline{\mathbf{R}}} r_{j, j-1}\right] \quad(\mathrm{A} .1) \\
\overline{\mathbf{F}}_{r 2}= & {\left[\frac{H_{n}^{(2)}\left(k_{\rho_{j}} \rho\right)}{n H_{n}^{(2)}\left(k_{\rho_{j}} \rho\right)} \overline{\mathbf{I}}+\frac{H_{n}^{(2)}\left(k_{\rho_{j}} a_{j}\right)}{H_{n}^{(2)}\left(k_{\rho_{j}} \rho\right)} \frac{J_{n}^{\prime}\left(k_{\rho_{j}} \rho\right)}{n J_{n}\left(k_{\rho_{j}} a_{j}\right)} \tilde{\overline{\mathbf{R}}} r_{j, j+1}\right] } \\
& \times \tilde{\overline{\mathbf{M}}}{ }_{j+}\left[\overline{\mathbf{I}}+\frac{J_{n}\left(k_{\rho_{j}} a_{j-1}\right)}{J_{n}\left(k_{\rho_{j}} \rho^{\prime}\right)} \frac{H_{n}^{(2)}\left(k_{\rho_{j}} \rho^{\prime}\right)}{H_{n}^{(2)}\left(k_{\rho_{j}} a_{j-1}\right)} \tilde{\overline{\mathbf{R}}} r_{j, j-1}\right]
\end{aligned}
$$

$$
\begin{aligned}
\overline{\mathbf{F}}_{r 3}= & {\left[\overline{\mathbf{I}}+\frac{J_{n}\left(k_{\rho_{j}} \rho\right)}{J_{n}\left(k_{\rho_{j}} a_{j}\right)} \frac{H_{n}^{(2)}\left(k_{\rho_{j}} a_{j}\right)}{H_{n}^{(2)}\left(k_{\rho_{j}} \rho\right)} \tilde{\overline{\mathbf{R}}} r_{j, j+1}\right] \tilde{\overline{\mathbf{M}}}{ }_{j+} } \\
& \times\left[\frac{J_{n}^{\prime}\left(k_{\rho_{j}} \rho^{\prime}\right)}{n J_{n}\left(k_{\rho_{j}} \rho^{\prime}\right)} \overline{\mathbf{I}}\right. \\
& \left.+\frac{J_{n}\left(k_{\rho_{j}} a_{j-1}\right)}{J_{n}\left(k_{\rho_{j}} \rho^{\prime}\right)} \frac{H_{n}^{\prime(2)}\left(k_{\rho_{j}} \rho^{\prime}\right)}{n H_{n}^{(2)}\left(k_{\rho_{j}} a_{j-1}\right)} \tilde{\mathbf{R}} r_{j, j-1}\right]
\end{aligned}
$$

$$
\begin{aligned}
\overline{\mathbf{F}}_{r 4}= & \frac{H_{n}^{\prime(2)}\left(k_{\rho_{j}} \rho\right)}{n H_{n}^{(2)}\left(k_{\rho_{j}} \rho\right)} \frac{J_{n}^{\prime}\left(k_{\rho_{j}} \rho^{\prime}\right)}{n J_{n}\left(k_{\rho_{j}} \rho^{\prime}\right)} \\
& \times\left[\overline{\mathbf{I}}+\frac{H_{n}^{(2)}\left(k_{\rho_{j}} a_{j}\right)}{H_{n}^{\prime(2)}\left(k_{\rho_{j}} \rho\right)} \frac{J_{n}^{\prime}\left(k_{\rho_{j}} \rho\right)}{J_{n}\left(k_{\rho_{j}} a_{j}\right)} \tilde{\mathbf{R}} r_{j, j+1}\right] \tilde{\overline{\mathbf{M}}}_{j+} \\
& \times\left[\overline{\mathbf{I}}+\frac{J_{n}\left(k_{\rho_{j}} a_{j-1}\right)}{J_{n}^{\prime}\left(k_{\rho_{j}} \rho^{\prime}\right)} \frac{H_{n}^{\prime}(2)\left(k_{\rho_{j}} \rho^{\prime}\right)}{H_{n}^{(2)}\left(k_{\rho_{j}} a_{j-1}\right)} \tilde{\mathbf{R}} r_{j, j-1}\right] .
\end{aligned}
$$

\section{REFERENCES}

[1] Y. L. Chow, J. J. Yang, D. F. Fang, and G. E. Howard, "A closed-form spatial Green's function for the thick microstrip substrate," IEEE Trans. Microwave Theory Tech., vol. 39, pp. 588-592, Mar. 1991.

[2] M. I. Aksun and R. Mittra, "Derivation of closed-form Green's functions for a general microstrip geometry," IEEE Trans. Microwave Theory Tech., vol. 40, pp. 2055-2062, Nov. 1992.

[3] G. Dural and M. I. Aksun, "Closed-form Green's functions for general sources and stratified media," IEEE Trans. Microwave Theory Tech., vol. 43, pp. 1545-1552, Jul. 1995.

[4] M. I. Aksun, "A robust approach for the derivation of closed-form Green's functions," IEEE Trans. Microwave Theory Tech., vol. 44, pp. 651-658, May 1996.

[5] M. I. Aksun and G. Dural, "Clarification of issues on the closed-form Green's functions in stratified media," IEEE Trans. Antennas Propag., vol. 53, pp. 3644-3653, Nov. 2005.

[6] J. R. Lovell and W. C. Chew, "Response of a point source in a multicylindrically layered medium," IEEE Trans. Geosci. Remote Sensing, vol. GRS-25, pp. 850-858, Nov. 1987.

[7] L. W. Pearson, "A construction of the fields radiated by z-directed point sources of current in the presence of a cylindrically layered obstacle," Radio Sci., vol. 21, pp. 559-569, 1986.

[8] K. Naishadham and L. B. Felsen, "Dispersion of waves guided along a cylindrical substrate-superstrate layered medium," IEEE Trans. Antennas Propag., vol. 41, pp. 304-313, Mar. 1993.

[9] Z. Xiang and Y. Lu, "Electromagnetic dyadic Greens function in cylindrically multilayered media," IEEE Trans. Microwave Theory Tech., vol. 44, pp. 614-621, Apr. 1996.

[10] P.-S. Kildal, S. Rengarajan, and A. Moldsvor, "Analysis of nearly cylindrical antennas and scattering problems using a spectrum of two-dimensional solutions," IEEE Trans. Antennas Propag., vol. 44, pp. 1183-1192, Aug. 1996.

[11] P.-S. Kildal, Z. Sipus, and M. Johansson, "Analysis of antennas on a curved multilayer structure by using the G1DMULT routine," in IEEE APS Int. Symp. URSI Radio Sci. Meeting Dig., Jul. 1997, vol. 3, pp. $1500-1503$

[12] W. C. Chew, Waves and Fields in Inhomogeneous Media. New York: Van Nostrand Reinhold, 1990.

[13] K.-L. Wong, Design of Nonplanar Microstrip Antennas and Transmission Lines. New York: Wiley, 1999.

[14] L. Josefsson and P. Persson, Conformal Array Antenna Theory and Design. New York: Wiley, 2006.

[15] C. Tokgöz, "Derivation of closed-form Green's functions for cylindrically stratified media," M.S. thesis, Dept. Elect. Electron. Eng., Middle East Technical Univ., Ankara, Turkey, Aug. 1997.

[16] C. Tokgöz and G. Dural, "Closed-form Green's functions for cylindrically stratified media," IEEE Trans. Microwave Theory Tech., vol. 48, pp. 40-49, Jan. 2000

[17] J. Sun, C.-F. Wang, L.-W. Li, and M.-S. Leong, "A complete set of spatial-domain dyadic Green's function components for cylindrically stratified media in fast computational form," J. Electromagn. Waves Appl., vol. 16, no. 11, pp. 1491-1509, 2002.

[18] R. C. Acar and G. Dural, "Comments on a complete set of spatialdomain dyadic Green's function components for cylindrically stratified media in fast computational form," J. Electromagn. Waves Appl., vol. 18, no. 10, pp. 1389-1394, 2004

[19] J. Sun, C.-F. Wang, L.-W. Li, and M.-S. Leong, "Reply to comments on a complete set of spatial-domain dyadic Green's function components for cylindrically stratified media in fast computational form," $J$. Electromagn. Waves Appl., vol. 18, no. 10, pp. 1395-1398, 2004.

[20] M. He and X. Xu, "Closed-form solutions for analysis of cylindrically conformal microstrip antennas with arbitrary radii," IEEE Trans. Antennas Propag., vol. 53, pp. 518-525, Jan. 2005. 
[21] J. Sun, C.-F. Wang, L.-W. Li, and M.-S. Leong, "Further improvement for fast computation of mixed potential Green's functions for cylindrically stratified media," IEEE Trans. Antennas Propag., vol. 52, pp. 3026-3036, Nov. 2004.

[22] R. C. Acar and G. Dural, "Complete set of closed-form Green's functions for cylindrically layered media," in Proc. IEEE AP-S Int. Symp. USNC/URSI Nat. Radio Sci. AMEREM Meetings, Jul. 2006.

[23] S. Karan, V. B. Ertürk, and A. Altintas, "Closed-form Green's function representations in cylindrically stratified media for method of moments applications," in 2006 Eur. Conf. Antennas Propag. (EuCAP 2006), Nov. 2006.

[24] Y. Hua and T. K. Sarkar, "Generalized pencil-of-function method for extracting poles of an EM system from its transient response," IEEE Trans. Antennas Propag., vol. 37, pp. 229-234, Feb. 1989.

[25] M. Abramowitz and I. A. Stegun, Handbook of Mathematical Functions with Formulas, Graphs, and Mathematical Tables. New York: Dover, 1970.

[26] M. Marin and P. H. Pathak, Calculation of the surface fields created by a current distribution on a coated circular cylinder Tech. Rep. 721565-1, Apr. 1989.

[27] V. B. Erturk and R. G. Rojas, "Efficient analysis of input impedance and mutual coupling of microstrip antennas mounted on large coated cylinders," IEEE Trans. Antennas Propag., vol. 51, pp. 739-749, Apr. 2003.

[28] T. N. Kaifas and J. N. Sahalos, "Analysis of printed antennas mounted on a coated circular cylinder of arbitrary size," IEEE Trans. Antennas Propag., vol. 54, pp. 2797-2807, Oct. 2006.

[29] V. B. Erturk and R. G. Rojas, "Paraxial space-domain formulation for surface fields on a large dielectric coated circular cylinder," IEEE Trans. Antennas Propag., vol. 50, pp. 1577-1587, Nov. 2002.

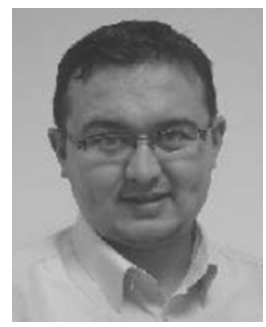

S. Karan received the B.S. and M.S. degrees from Bilkent University, Ankara, Turkey, in 2003 and 2006, respectively, where he is currently pursuing the Ph.D. degree in the Electrical and Electronics Engineering Department.

He has been with Aselsan Electronics Inc., Ankara, as an RF Antenna Engineer since 2003. His research interests include application of numerical methods to radiation and mutual coupling problems associated with cylindrical structures.

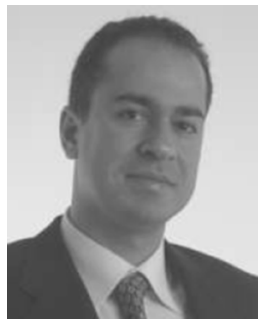

V. B. Ertürk (M'00) received the B.S. degree in electrical engineering from the Middle East Technical University, Ankara, Turkey, in 1993 and the M.S. and Ph.D. degrees from The Ohio State University, Columbus, in 1996 and 2000, respectively.

$\mathrm{He}$ is currently an Associate Professor with the Electrical and Electronics Engineering Department, Bilkent University, Ankara. His research interests include the analysis and design of planar and conformal arrays, active integrated antennas, profiles, and metamaterials. scattering from and propagation over large terrain

Dr. Ertürk was Secretary/Treasurer of the IEEE Turkey Section and the Turkey Chapter of the IEEE Antennas and Propagation, Microwave Theory and Techniques, Electron Devices, and Electromagnetic Compatibility societies. He received the 2005 URSI Young Scientist and 2007 Turkish Academy of Sciences Distinguished Young Scientist awards.

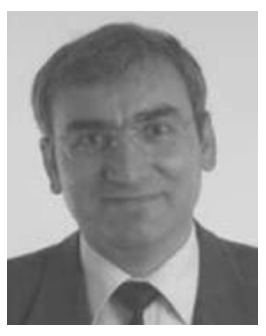

A. Altintas ( $\left.\mathrm{S}^{\prime} 82-\mathrm{M}^{\prime} 87-\mathrm{SM}^{\prime} 93\right)$ received the B.S. and M.S. degrees from the Middle East Technical University, Ankara, Turkey, in 1979 and 1981, respectively, and the Ph.D. degree from The Ohio State University (OSU), Columbus, in 1986.

From 1981 to 1987, he was with the ElectroScience Laboratory, OSU. He is currently Professor and Chair of Electrical Engineering at Bilkent University, Ankara. He has been a Research Fellow and Guest Professor at Australian National University, Canberra, Australia; Tokyo Institute of Technology, Japan; Technical University of Munich, Germany; and Concordia University, Montreal, PQ, Canada. His research interests include high-frequency and numerical techniques in electromagnetic scattering and diffraction, propagation modeling and simulation, and fiber and integrated optics. He has served on many university committees and was Associate Provost of Bilkent University for 1995-1998. He is the National Chair of URSI Commission B.

Dr. Altintas is a member of Sigma Xi and Phi Kappa Phi. He is a Fulbright Scholar and an Alexander von Humboldt Fellow. He was Chair of the IEEE Turkey Section for 1991-1993 and 1995-1997. He is the Founder and first Chair of the IEEE AP/MTT Chapter in the Turkey Section. He received the ElectroScience Laboratory Outstanding Dissertation Award in 1986; the IEEE 1991 Outstanding Student Branch Counselor Award; the 1991 Research Award from the Prof. Mustafa N. Parlar Foundation, METU; and the Young Scientist Award from the Scientific and Technical Research Council of Turkey (Tubitak) in 1996. He received an IEEE Third Millennium Medal. 